\title{
Telekommunikationsnetze in Deutschland - mit einem öffentlichen Unternehmen ausbauen
}

\author{
Die Debatte um den Ausbau von Mobilfunk- und Glasfasernetzen in Deutschland bedarf einer \\ Objektivierung. Statt Ängste zu schüren, gilt es nüchtern zu überprüfen, ob die Privatisierung \\ der physischen Infrastruktur vor rund 25 Jahren sowie die getrennte Betrachtung von \\ Glasfaser- und Mobilfunkausbau noch zeitgemäß ist, insbesondere wenn der Zugang \\ zum Netz zunehmend unter dem Aspekt der Daseinsvorsorge bewertet wird. Vor diesem \\ Hintergrund bedarf es einer ganzheitlichen Ausbauplanung für Glasfaser und Mobilfunk, \\ die Versorgungsziele losgelöst vom Übertragungsweg mit Blick auf die tatsächliche \\ Nutzung flächenbezogen definiert. Am effizientesten lässt sich dies durch eine öffentliche \\ Infrastrukturgesellschaft umsetzen, die keine Änderungen im bestehenden Wettbewerbs- und \\ Beihilferecht erfordern würde.
}

\begin{abstract}
Infrastrukturdebatten werden in Deutschland selten rational geführt. Ein Instrument der Emotionalisierung ist dabei der Verweis auf vermeintlich schlechte Rankings im internationalen Vergleich und den Verlust der Wettbewerbsfähigkeit. Um die Dramatik zu unterstreichen, wird dann gerne auf Länder Bezug genommen, mit denen sich Deutschland sonst eher selten vergleicht. „Beim Handynetz schlechter als Albanien" betitelte etwa die Welt (O. V., 2018) einen Bericht über eine Studie zur Mobilfunkabdeckung in Europa. Das eigentliche Anliegen der Studie - ein Plädoyer für nationales Roaming, also die gemeinsame Nutzung eines Mobilfunkstandortes durch mehrere Mobilfunkanbieter - war dann zwar in der Diskussion

(C) Der/die Autor(en) 2020. Open Access: Dieser Artikel wird unter der Creative Commons Namensnennung 4.0 International Lizenz (https:// creativecommons.org/licenses/by/4.0/deed.de) veröffentlicht.

Open Access wird durch die ZBW - Leibniz-Informationszentrum Wirtschaft gefördert.

* Der Text gibt ausschließlich die persönliche Meinung der Autoren wieder.
\end{abstract}

Dr. Cordelius Ilgmann ist Abteilungsleiter im Thüringer Ministerium für Wirtschaft, Wissenschaft und Digitale Gesellschaft.

André Störr ist Kompetenzteamleiter in der Abteilung Kompetenzzentrum Verwaltung 4.0 des Thüringer Landesverwaltungsamtes. kein Thema, aber das Alarmzeichen war gesetzt. Ähnlich verläuft die Debatte zum Ausbau des Glasfasernetzes in Deutschland. Auch hier überwiegt der Tenor, die Bundesrepublik laufe Gefahr, im internationalen Standortwettbewerb abgehängt zu werden (Scheuer, 2019).

Es soll nicht bestritten werden, dass im Bereich der Telekommunikationsdienste sowohl bei der kabelgebundenen Versorgung als auch bei der Mobilfunkversorgung in Deutschland noch Lücken bestehen. Auch wenn diese nur einen kleinen Teil der Haushalte betreffen, zeigen sich regionale Unterschiede, die dem Problem eine Brisanz geben. Denn der unzureichende Zugang zu digitalen Infrastrukturen beeinträchtigt weniger wirtschaftlich prosperierende, zumeist urbane Gebiete, sondern ländliche Regionen. Damit erhalten Zustand und Ausbau der digitalen Infrastrukturen eine zusätzliche politische Dimension im Hinblick auf die Sicherstellung gleichwertiger Lebensverhältnisse.

Vereinfacht lässt sich die Debatte auf zwei Lager reduzieren: Die einen verweisen auf den inzwischen im Grundgesetz und in den Vorgaben der EU angelegten Wettbewerb im Bereich der Telekommunikationsdienstleistungen, der es weitgehend dem Markt überlässt eine Versorgung sicherzustellen. Als Ausgangspunkt dient Art. $87 \mathrm{f} \mathrm{GG}$, der im Rahmen der Postreform 1992 geschaffen wurde und ein Primat des Marktes vorsieht. Innerhalb der Werteordnung des Grundgesetzes ist diese Norm übrigens eine Besonderheit, da die Verfassung in Bezug auf die Wirtschaftsordnung eigentlich neutral ausgestaltet ist. Dass nicht jede "Milchkanne" versorgt wird, ist nach diesem Verständnis Ausdruck marktwirtschaftlicher Effizienz und einer ange- 
messenen Reaktion auf eine geringe Nachfrage. Die gegenteilige Auffassung verweist auf die Bedeutung der digitalen Infrastruktur für die Zukunft des ländlichen Raums und begreift Breitbandverbindung und Mobilfunkempfang zu allererst als notwendige Daseinsvorsorge, die unabhängig vom Markt durch den Staat zu erbringen ist ( $z$. B. Grüne Bundestagsfraktion; 2020). Auch diese Haltung nimmt Bezug auf Art. 87 f GG, stellt aber den dort auch formulierten Gewährleistungsauftrag des Bundes in den Vordergrund.

Die beiden Positionen verdeutlichen, dass sich eine Vorgabe zum Umfang des Netzausbaus nicht einfach aus den Rechtsgrundlagen ableiten lässt. Vielmehr lohnt sich die Frage, ob die vor 25 Jahren getroffenen Annahmen zum Design des Telekommunikationsmarkts heute noch Bestand haben können. So wurde einem liberalisierten Markt im letzten Viertel des 20. Jahrhunderts die Fähigkeit zugemessen, auf den steten Wandel und den raschen technologischen Fortschritt im Bereich der Telekommunikation effizienter reagieren zu können als ein staatliches Monopol. Gleichzeitig sollte die Privatisierung helfen, die notwendigen privaten und öffentlichen Mittel für den Aufund Ausbau neuer Telekommunikationsangebote zu mobilisieren (Europäische Kommission, 1995). Damals gab es für jeden einzelnen Typus von Telekommunikationsdienst eigene Übertragungslinien und es galt als fortschrittlich, das Netz der Zukunft als konvergent zu denken.

Beide damalige Grundannahmen treffen heute in der Form nicht mehr zu. Zum einen ist die technologische Unsicherheit in Bezug auf die physische Infrastruktur verschwunden: Kabelgebundenes und mobiles Internet werden in absehbarer Zeit zu einem konvergenten Netz zusammenwachsen, dessen Rückgrat ein flächendeckendes Glasfasernetz bis in die Gebäude und an die Mobilfunkstandorte sein wird. Zum anderen deutet sich an, dass die öffentliche Hand Netzinfrastrukturen mit dem Charakter von natürlichen Monopolen volkswirtschaftlich effizienter errichten und finanzieren kann. Denn beim derzeitigen privatwirtschaftlichen Ausbau ist zu erkennen, dass einerseits in dichter besiedelten Gebieten redundante physische Infrastrukturen entstehen, während gerade im ländlichen Raum der Ausbau in Teilen oder gar gänzlich unterbleibt. Vor allem aber zeichnet sich ab, dass sich die seit den 1990er Jahren breite Netzprivatisierung im Bereich der Fernsehkabel- und Telefonnetze hin zu wenigen Anbietern konzentrierte und damit zunehmend oligopolartige Strukturen entwickelt hat.

Der Bund, dem das Grundgesetz den Gewährleistungsauftrag zur Sicherung eines Zugangs zu digitalen Infrastrukturen zuweist, agiert vor diesem Hintergrund in den letzten Jahren widersprüchlich. So hält er am überholten Konzept des Infrastrukturwettbewerbs fest, versucht aber gleichzeitig ordnungspolitische Defizite mit Milliarden an Fördermitteln für den Breitbandausbau sowie neuerdings für Mobilfunkmasten zu kompensieren. Statt einer Finanzierung durch den Markt, wie es bei der Privatisierung der Telekommunikationsdienstleistungen angestrebt war, werden die privaten Kosten des Ausbaus der Telekommunikationswege zu nicht unerheblichen Teilen auf die Steuerzahler verlagert, inzwischen mit fast 10 Mrd. Euro nur für die Schließung bestehender Versorgungslücken im Breitband. ${ }^{1}$ Gleichzeitig hat der Bund die Verantwortung für die Erfüllung seines Gewährleistungsauftrags derzeit an die kommunalen Gebietskörperschaften delegiert. Dies führt nicht nur zu einer ungerechtfertigten Belastung von Städten und Gemeinden, sondern auch zu einem Flickenteppich an Lösungen und Zuständigkeiten. Wenn aber mittlerweile weitgehend Konsens ist, dass zur Sicherstellung künftiger Telekommunikationsbedarfe Glasfaser- und darauf aufbauend Mobilfunknetze flächendeckend errichtet werden müssen, drängt sich die Frage auf, wieso Bund und Länder an diesem komplexen Verfahren festhalten, statt durch einfachere Alternativen den Ausbau erheblich zu beschleunigen und Versorgungssicherheit zu schaffen.

\section{Verlässliche Daten erforderlich}

Dass die aktuelle Debatte mehr emotional als rational geführt wird, hat wesentlich auch mit der fehlenden Verfügbarkeit von verlässlichen Daten zu tun. So existiert im Mobilfunk nach wie vor keine systematische Überprüfung der Ist-Versorgung im Sinne der Versorgungsgüte (Quality of Service) unter realistischen Bedingungen. Messverfahren zur Überprüfung von Versorgungsauflagen beziehen sich bisher maßgeblich auf technische Parameter. Der tatsächliche Kundennutzen unter realistischen Bedingungen wird dagegen nicht erfasst. So bleiben Auswirkungen von Schirmungen durch Gebäudedämpfung bei der Beschreibung der Versorgungslage ebenso unberücksichtigt wie die Geschwindigkeit von Fahrzeugen und Zügen, aus denen heraus Netzverbindungen erfolgen. Auch Funklöcher werden nicht systematisch durch Messungen aufgespürt, sondern durch einen Erhebungsmix aus gelegentlichen Kontrollmessungen, Mitteilungen zu Funklöchern durch die Bundesländer und in jüngster Zeit auch durch die Funkloch-App der Bundesnetzagentur, einer Anwendung für mobile Endgeräte der Netznutzer.

Die Studie zu 5G National Roaming (P3 communications, 2018), die im Auftrag der Bundestagsfraktion von Bündnis

1 Dabei wird angenommen, dass der Bund bislang zwischen Mitte 2015 bis Anfang 2020 fast 6 Mrd. Euro mit einem durchschnittlichen Fördersatz von ca. $60 \%$ bewilligt hat, sodass die übrigen $4 \mathrm{Mrd}$. Euro bzw. $40 \%$ durch Länder und Kommunen erbracht werden. 
90/Die Grünen entstanden ist, verdeutlicht die methodischen Schwächen bestehender Erhebungen. ${ }^{2}$ Mit Hilfe einer Android-App, die auf weltweit 190 Mio. Geräte installiert war und täglich etwa 1,5 Billionen Datensätze lieferte, wurden im Zeitraum Juli bis September 2018 Daten erhoben. Aus dieser Crowdsourcing-Messung wurde ein internationaler Vergleich verschiedener Netzbetreiber vorgenommen. Demnach bietet die Deutsche Telekom nur bei rund $75 \%$ der erhobenen Datensätze eine 4G-Funkversorgung. Zum Vergleich werden die Niederlande, Belgien und die Schweiz angeführt, wo bereits fast alle Netzbetreiber einen Anteil am Mobilfunkstandard Long Term Evolution (LTE) von mehr als $90 \%$ anbieten. Noch schlechter schneiden alle drei deutschen Netzbetreiber beim Vergleich der durchschnittlich genutzten Datenrate ab: Mit weniger als $5 \mathrm{Mbit} / \mathrm{s}$ belegen sie die letzten Plätze - ein vermeintlich desaströses Ergebnis, da die Telekom Albanien mit 9,4 Mbit/s eine ungefähr doppelt so hohe durchschnittliche Datenrate aufweist.

Allerdings macht die Studie keine Aussage darüber, ob die betrachtete Stichprobe repräsentativ ist. Dabei weisen gerade Messungen mit Crowdsourcing-Methoden eine hohe Varianz auf. So hat eine alternative Studie zur Verfügbarkeit von 3G und 4G eine durchschnittliche Downloadgeschwindigkeit von rund $30 \mathrm{Mbit} / \mathrm{s}$ erbracht - rund das 6-fache des Wertes der P3-Studie (Open Signal, 2018). Hinzu kommt, dass die genutzte Datenrate entscheidend vom jeweiligen Kundenvertrag abhängt. Wer aus Kostengründen als Verbraucher auf eine optimale Versorgung verzichtet, beeinflusst damit die Aussagen zur Netzqualität. So sind in Deutschland Mobilfunk-Discounter mit rund einem Viertel Marktanteil so stark wie nirgendwo sonst in Europa vertreten. Diese schließen allerdings in ihren Vertragsbedingungen vielfach die 4G-Nutzung aus. Nach einer Erhebung der Bundesnetzagentur (2018) sind nur etwa die Hälfte der SIMKarten in Deutschland LTE-fähig. Die vermeintlich schlechten Ergebnisse der Studie verweisen dann weniger auf eine per se schlechte Mobilfunkinfrastruktur, als auf eine geringe Nachfrage nach schnellem mobilen Internet (Rizzato, 2019).

Hierzu passen Daten der OECD (2018) zu den relativen Preisen von Mobilfunkleistungen. ${ }^{3}$ Aus diesen ergibt sich,

2 Dies gilt auch für die Studie im Auftrag des Bundesministeriums für Verkehr und digitale Infrastruktur (WIK und Umlaut, 2019), auf deren Grundlage die in der Öffentlichkeit kursierenden 5.700 weiße Flecken im Mobilfunk prognostiziert wurden. Die Studie beruht auf Crowdsourcing-Messung, wobei für etwa $1 / 3$ der Fläche nicht genügend Daten zur Verfügung standen.

3 Nach kaufkraftbereinigten Daten für Mai/Juni 2017 der OECD (2018) zahlen in Deutschland geringe Nutzer, definiert als 100 Anrufe und 500 Mbit Datennutzung pro Monat, rund 12,50 US-\$ (OECD-Durchschnitt ca. 22,50 US-\$), durchschnittliche Nutzer, definiert mit 300 Anrufen und 1 Gigabit Datennutzung, zahlen monatlich im Schnitt 24,65 US-\$ (OECD-Durchschnitt ca. 29,80 US-\$) und starke Nutzer, definiert als 900 Anrufe sowie 2 Gigabit Datennutzung, zahlen monatlich im Schnitt 37,50 US-\$ (OECD-Durchschnitt ca. 36,80 US-\$). dass Mobilfunkdienstleistungen im OECD-Vergleich in Deutschland verhältnismäßig günstig sind. Der Wettbewerb zeigt bei den Verbraucherpreisen also eine positive Wirkung. Gleichzeitig zahlen die Mobilfunknetzbetreiber vergleichsweise hohe Preise bei Frequenzversteigerungen. So wurden bei der 2019 abgeschlossenen Frequenzauktion im Deutschland von den Betreibern ca. 0,22 Euro/ $\mathrm{MHz/potenziellen} \mathrm{Kunden} \mathrm{bezahlt,} \mathrm{ein} \mathrm{im} \mathrm{europäischen}$ Bereich eher überdurchschnittlicher Wert (GSMA Spectrum, 2017). Daraus lässt sich die These ableiten, dass der derzeit als unzureichend wahrgenommene Ausbauzustand auch durch eine Kombination von strengem Wettbewerb auf der Ebene der Dienste in Verbindung mit vergleichsweise hohen Frequenzkosten verursacht wurde.

\section{Teil der Daseinsvorsorge}

Tatsächlich ist in Deutschland nach wie vor die Erzielung möglichst hoher staatlicher Einnahmen ein maßgeblicher Zielindikator bei der Zuweisung von Funkfrequenzen an Marktteilnehmer. Diese Zielsetzung stammt indes noch aus einer Epoche, in der Mobilfunk als ein Zusatzgut angesehen wurde, das eher in den Bereich der Freizeitoder Luxusgüter fiel. ${ }^{4}$ Spätestens mit der Einführung von Smartphones 2007 und der damit wirklich sinnvollen Nutzbarkeit des bereits 2000 eingeführten Übertragungsstandards der 3. Generation hat sich aber ein Wandel in den Lebensgewohnheiten vollzogen. Mobile Formen der Kommunikation mit und durch digitale Medien gehören für den weit überwiegenden Teil der Bevölkerung zum Alltag. ${ }^{5}$

Mit guten Gründen wird deshalb längst dafür geworben, auch oder womöglich sogar gerade die ortsungebundene Telekommunikation und den ortsungebundenen Informationszugang als Teil der Daseinsvorsorge anzusehen (Grüne Bundestagsfraktion, 2020), soweit an diesen wegen seiner Begriffsherkunft (Forsthoff, 1938) nicht unproblematischen Begriff angeknüpft werden soll. Bislang interpretiert der Bund, auch vor dem Hintergrund einschlägiger EU-Gesetzgebung, den im Artikel 87 f GG normierten Gewährleistungsauftrag sehr restriktiv. So ist zwar einfachgesetzlich ein sogenannter Universaldienst vorgegeben. Beschrieben ist damit ein Mindestmaß an Diensten der Fernkommunikation und -information, die für alle verfügbar sein müssen. Jedoch ist dieser Univer-

4 Schon bei der ersten Postreform 1989 wurden lediglich die kabelgebundenen Telekommunikationsnetze und -dienste als staatliches Monopol zurückbehalten, der Bereich des Mobilfunks dagegen sogleich für den Wettbewerb freigegeben. Das führte im gleichen Jahr zur Etablierung des (privaten) D2-Netzes und 1990 zum Eintritt der Mannesmann AG in den Telekommunikationsmarkt.

5 So wird der Anteil der Smartphonenutzer an der Gesamtbevölkerung für Deutschland 2018 bereits mit $81 \%$ angegeben (statista, 2020). 
saldienst nach geltender Rechtslage auf Sprachtelefonie und einen einfachen Internetzugang an einem festen Ort beschränkt. Mobile Telekommunikationsverbindungen fallen derzeit nicht darunter.

Nichtsdestoweniger scheint der Bund einen staatlichen Auftrag zur Sicherstellung eines flächendeckenden Mobilfunknetzes inzwischen anzuerkennen. Jedoch sind die Maßnahmen, die in der Mobilfunkstrategie des Bundes als Handlungsoptionen vorgestellt werden, nicht frei von Ironie. Zum einen wird über ein eigenständiges Förderprogramm nachgedacht, das aus den Einnahmen der Frequenzvergaben gespeist werden soll - ein besonderes Modell der Kreislaufwirtschaft. Zum anderen brachte die CDU/CSU-Fraktion eine Mobilfunk-Infrastrukturgesellschaft (MIG) ins Gespräch, die selbst insbesondere Funkmasten für den Mobilfunk errichten soll. Allerdings ist diese in der politischen Debatte in ihren möglichen Aufgaben bereits zu einer nur koordinierenden Stelle zusammengeschrumpft, sodass im Zentrum der derzeitigen Überlegungen die Konzeption eines eigenständigen Mobilfunkförderprogramms steht (BMVI, 2019a). Insofern ist die Mobilfunkstrategie als verpasste Chancen zu bewerten, den strategischen Rahmen neu zu denken. Hierzu hätte nicht nur eine konsequente Ausrichtung des Ausbaus an den tatsächlichen Bedarfen sowie die Erhebung der entsprechenden Daten aus Nutzersicht gehört, sondern auch eine kritische Auseinandersetzung mit der tatsächlichen und der avisierten Verfügbarkeit des Mobilfunks im Kontext der flächendeckenden Versorgung, gerade im Hinblick auf die Gleichwertigkeit der Lebensverhältnisse.

\section{Glasfaser- und Mobilfunkausbau ganzheitlich denken}

Im Jahr 2018 waren erst 3,2\% der kabelgebundenen Hausanschlüsse in Deutschland direkt durch Glasfaser versorgt (OECD, 2018). Dies ist aber per se kein Grund zur Besorgnis. Denn es spricht viel dafür, dass der schleppende Ausbau der Glasfaserinfrastruktur auch auf die gegenwärtig für die Nachfrage ausreichende Versorgung durch Bestandsinfrastrukturen zurückzuführen ist, die gerade in Deutschland vergleichsweise leistungsfähig sind (llgmann, 2019). Die Verteilung bestehender unteroder gar unversorgter Gebiete zeigt aber - analog zur Mobilfunkversorgung - eine signifikante Benachteiligung des ländlichen Raums. Insofern ist es unzutreffend zu behaupten, dass Deutschland im internationalen Vergleich abgehängt sei. Vielmehr besteht die konkrete Gefahr, dass mit der zunehmenden Bedeutung digitaler Infrastrukturen die Lebensverhältnisse zwischen Stadt und Land weiter auseinanderdriften.

Allerdings ist das Ungleichgewicht im Ausbau zwischen dem städtischen und ländlichen Raum kein Naturgesetz.
Vielmehr ist es die erwartbare Folge der Entscheidung, die Erstellung der physischen „passiven“ Infrastruktur aus Rohren und Glasfaserleitungen - mit dem Charakter eines natürlichen Monopols - allein dem Markt zu überlassen, anstatt den Wettbewerb auf die Erbringung der Dienste zu beschränken. Nun droht ausgerechnet die vor einer Generation erfolgte Privatisierung für den Staatshaushalt teuer zu werden, da jetzt das Kupfernetz mit Glasfaser überbaut werden muss. Da der Netzausbau dem Wettbewerb überlassen wird, besteht für den Staat als Gewährleistungsträger derzeit keine Möglichkeit, die Ertragsverteilungen aus urbanen Gebieten mit wirtschaftlich betreibbarer Infrastruktur hin zu ländlichen Räumen mit besonders hohen Erschließungsaufwänden umzuverteilen. Stattdessen werden Gewinne aus den urbanen Räumen von privaten Telekommunikationsunternehmen zu großen Teilen darauf verwendet, dort Doppelstrukturen aufzubauen, um eigene Übertragungswege zur Sicherung der strategischen Wettbewerbsposition zu nutzen. Unwirtschaftliche Aufwände sollen dann aus den Staatshaushalten kompensiert werden.

Jedoch bleibt trotz des inzwischen geschaffenen Ausbauanreizes durch Fördermittel aus öffentlichen Haushalten die Schließung von Versorgungslücken deutlich hinter den Erwartungen zurück. Als das aktuelle Förderprogramm des Bundes 2015 eingerichtet wurde, war ein Abschluss der Ausbaumaßnahmen bis 2018 vorgesehen. Allerdings flossen von den bereitgestellten Milliardensummen bis dahin lediglich 44 Mio. Euro auch tatsächlich ab (Antwort auf die Kleine Anfrage, 2018). Angesichts von Zuwendungsverträgen, die in vielen Fällen einen Umsetzungszeitraum von vier Jahren vorsehen, ist ein Abschluss der Verfahren erst 2024 realistisch.

Ein wesentlicher Grund für die lange Verfahrensdauer ist die Entscheidung des Bundes, die Förderung an die Kommunen zu richten. So ist für die Subventionsgewährung an ein Telekommunikationsunternehmen im vorherrschenden Wirtschaftlichkeitslückenmodell in der Regel jeweils eine Zuwendungsentscheidung von Bund, Bundesland und Kommune notwendig. Trotz aller lobenswerten Bemühungen der zuständigen Bewilligungsbehörden ist dieses Verfahren mit drei (!) formell unabhängigen, aber miteinander verwobenen Verwaltungsentscheidungen alles andere als ein einfaches, unbürokratisches Verfahren - von der ungerechtfertigten administrativen und finanziellen Belastung gerade strukturschwächerer Städte und Gemeinden im ländlichen Raum ganz zu Schweigen.

Nichtsdestoweniger wurde inzwischen durch die Bundespolitik ein neues Ziel vorgegeben, bis 2025 eine flächendeckende Gigabitversorgung erreichen zu wollen. Selbst ungeachtet des Umstands, dass damit erneut nur 
eine Datenrate und kein Infrastrukturziel „Glasfaser“ vorgegeben wird, zeigt die Bundespolitik in Bezug auf den Netzausbau zu wenig Bereitschaft zu notwendigen Veränderungen und zur realistischen Zielsetzung. So zeichnet sich ab, dass erneut der kabelgebundene Ausbau und der Ausbau der Mobilfunkversorgung weitgehend losgelöst voneinander betrachtet werden. Dabei sind die Zeiten längst vorbei, in denen Funknetze und Kabelnetze getrennte Übertragungswege bildeten. Schon heute besteht eine Konvergenz in Bezug auf die Backhaul-Verbindungen, mit denen im Mobilfunk die Basisstationen an die übergeordneten Netzwerkkomponenten angeschlossen werden. Diese Glasfaserverbindungen stellen sicher, dass die Daten zwischen den Stationen zuverlässig und in hohen Raten abtransportiert werden können und nicht zuletzt auch die Latenzzeiten, die unvermeidbaren Verzögerungen zwischen Absenden und Empfangen von Signalen über große Distanzen, so gering wie möglich gehalten werden können. Denn die Lichtwellenleitung in Glasfasern ist erheblich schneller als die Ausbreitung von Funkwellen im Luftraum. Spätestens für den Mobilfunk der 5. Generation (5G) wird es unvermeidbar sein, für diese Verbindungen zwischen den Funkstationen, die mit den Endgeräten kommunizieren, Glasfaserleitungen zu nutzen, um die an die neue Netzgeneration gerichteten Erwartungen tatsächlich einlösen zu können.

Es ist damit zu befürchten, dass sich das Stadt-LandGefälle gerade im Mobilfunk verschärfen wird. Denn während in urbanen Zentren ausreichend Glasfaserleitungen zur kostengünstigen Nutzung für Mobilfunkstationen zur Verfügung stehen, droht im ländlichen Raum eine erheblich eingeschränkte Servicequalität des Netzes. So wird zwar jede Milchkanne Signale absetzen können, wenn sie smart genug dazu ist. Sie wird aber auf eine Antwort deutlich länger als ein smarter Kühlschrank im urbanen Loft warten müssen. Und mobiles Streamen von Videodateien wird auch künftig eher nicht zu den Freizeitaktivitäten der Menschen jenseits urbaner Zentren gehören.

Zwar wurde in die Förderrichtlinie des Bundes (BMVI, 2019b) eine Förderung zur Herstellung konvergenter Netze aufgenommen. Demnach soll (bzw. kann) der Netzausbau auch genutzt werden, um Mobilfunkstandorte an das neue Netz anzuschließen. Jedoch muss ein solcher Anschluss nicht zwingend erfolgen und es wird auch keine Planung eines Mobilfunknetzes im Ausbaugebiet verlangt. Zudem werden weder die Errichtung von Masten noch die über den Telekommunikationsanschluss hinausgehenden Erschließungsarbeiten vom Fördertatbestand erfasst. Es ist schon fraglich, ob sich ein Mobilfunkanbieter und ein anderes Telekommunikationsunternehmen, das eine Ausbauförderung erhalten hat, den Graben zur
Verlegung von Leitungen teilen dürfen oder ob dies aus beihilferechtlichen Gründen unterbleiben muss. ${ }^{6}$

Trotz des unmittelbaren sachlichen Zusammenhangs werden die jeweiligen Politikansätze in beiden Feldern noch immer unterschiedlich formuliert. Während der Glasfaserausbau durch milliardenschwere Förderprogramme in einem ansonsten weitestgehend liberalisierten Markt befördert werden soll, wird der Netzausbau im Mobilfunk durch Versorgungsauflagen bei der Zuweisung von Frequenzen gesteuert. Diese Trennung aufzuheben und Mobilfunk und Glasfaser künftig ganzheitlich zu planen und zu errichten, ist aber eine notwendige Bedingung, um den Ausbau der digitalen Infrastruktur effizienter zu gestalten.

\section{Zukunftsfähige Politik des Netzausbaus}

Unter dem Aspekt der konvergenten Nutzung und der damit notwendigen ganzheitlichen Netzplanung wäre es in einem ersten Schritt sinnvoll, Versorgungsziele mit Blick auf die Nutzung losgelöst vom Übertragungsweg flächenbezogen zu definieren. Denn zum einen kann ein leistungsfähiges Mobilfunknetz schon nach aktuellem Stand der Technik die Versorgung mit hochleistungsfähigen Breitbanddiensten in ähnlicher Qualität sicherstellen wie die verfügbaren kabelgebundenen Netze. Zum anderen verspricht der Mobilfunkstandard der nächsten Generation auch die Möglichkeit von Datenraten im Gigabitbereich, wie es als Versorgungsziel für die Mitte des aktuellen Jahrzehnts vorgegeben ist. Eine flächenbezogene statt einer haushaltsbezogenen Netzplanung würde somit den Verbrauchergewohnheiten der Gegenwart entsprechen, wonach der Unterschied zwischen mobiler und stationärer Nutzung zunehmend verschwimmt.

Der Schwerpunkt der Ausbauplanung muss daher - im Sinne der Sicherung gleichwertiger Lebensverhältnisse auf einer angemessenen Versorgungsqualität liegen. Dies erlaubt durchaus, in Bezug auf die einzelnen technischen Parameter, die mit der Datenübertragung zusammenhängen (Datenrate, Latenz, Fehlerquote), gebietsspezifische Ziele, insbesondere bei wenigen Teilnehmern in schwieriger Topographie, zu setzen. Für die Versorgung eines Siedlungsgebietes können auch grundsätzlich andere Schwerpunkte gesetzt werden als etwa entlang einer Landstraße oder eines Höhenwanderwegs. Entscheidend ist, dass dann aber für die so gesetzten Versorgungsziele konsequent die infrastrukturellen Voraussetzungen ge-

6 Zwar kann auch ein Mobilfunknetz ein Next-Generation-Access (NGA)Netz sein, dessen Förderung nach der NGA-Rahmenregelung eine zulässige Beihilfe darstellen kann. Fraglich ist aber, ob ein paralleler Ausbau eines kabelgebundenen und eines Mobilfunknetzes in einem unversorgten Gebiet durch Förderung zulässig ist, da dann zwei NGA-Netze entstehen und in der Programmlogik eine Überversorgung entstünde. 
schaffen werden. Die Vorrausetzung hierfür ist ein Glasfasernetz bis in die Gebäude und an die Mobilfunkstandorte. Allerdings meint flächendeckend im Sinne eines konvergenten Netzes nicht die Erschließung jedes einzelnen Grundstücks im Bundesgebiet, sondern ein Netzausbau, der eine raumbezogene Absicherung von gigabitfähigen Datenverbindungen erlaubt. Ein solcher Netzausbau hat gegenüber dem derzeitigen Modell den Vorteil einer deutlich besseren Effizienz sowohl in Bezug auf Planung und Ausbaukosten als auch auf die Netzabdeckung.

Allerdings verharrt der Bund - wie die meisten Bundesländer auch - in seiner Logik der getrennten Betrachtung mobiler und kabelgebundener Netze. Neben das bestehende Förderprogramm für den kabelgebundenen Breitbandausbau soll ein eigenes Förderprogramm für den Mobilfunk treten, von dem indes bisher wenig mehr sicher ist als die veranschlagte Fördersumme in Höhe von 1,1 Mrd. Euro. Die aktuelle Mobilfunkstrategie des Bundes gibt lediglich die „Konzeption, Abstimmung und Notifizierung einer Förderkulisse" vor und nennt als Zeitpunkt der Umsetzung „ab sofort“ (BMVI, 2019a, 42).

Aus den bisherigen Ankündigungen des Bundes lässt sich immerhin erkennen, dass der Mobilfunkausbau nur in Gebieten gefördert werden soll, in denen keine privaten Ausbaupläne bestehen und in denen kein Ausbau aufgrund von Versorgungsauflagen sowie aufgrund der vertraglichen Ausbauverpflichtungen der Netzbetreiber (Vereinbarung mit dem Bund vom 5. September 2019) geplant ist. Aus den Erfahrungen des bisherigen Breitbandförderprogramms lässt sich bereits heute vorhersagen, dass Abgrenzungsprobleme vorprogrammiert sind. Zugleich deutet sich an, dass der Bund den Fehler wiederholt, die Kommunen für die Umsetzung des Förderprogramms in die Verantwortung zu nehmen. Diese sollen dann nicht nur die weißen Flecken ermitteln, sondern auch die Zuwendung durch wettbewerbliche Ausschreibungen an die Unternehmen vergeben. Damit entsteht wieder ein mehrstufiges Verfahren, das einen administrativen Vorlauf von mehreren Jahren provoziert.

Zwar sollen die Kommunen „so weitgehend wie möglich“ entlastet werden, indem der MIG eine starke Rolle bei der Abwicklung des Förderprogramms, zur Planungsunterstützung, zur Bereitstellung von (Bundes-)Liegenschaften sowie zur Akzeptanzsteigerung des Mobilfunkausbaus zukommen soll. Doch bleibt schon die Abgrenzung der Aufgaben der MIG von denen anderer, mit dem Ausbau der digitalen Infrastruktur betrauten Institutionen offen. Vor allem aber wirkt das sich abzeichnende Vorgehen des Bundes wie der abermalige Versuch, die Vollzugslasten entgegen der Vorgabe aus Art. 85 Abs. 1 S. 2 GG auf kommunale Hoheitsträger abzuwälzen und zur Erfüllung eines Gewährleistungsauftrags des Bundes mittelbar in die Haushalte von Ländern und Kommunen einzugreifen.

\section{Ein dreistufiges Modell}

Dabei gibt es eine sofort umsetzbare Alternative, um eine effiziente Planung und den Ausbau konvergenter Netze auf der Basis definierter Versorgungsziele einzuleiten, ohne dass in den Infrastrukturbestand eingegriffen wird. Der Schlüssel dazu liegt im Betreibermodell. Bei diesem Modell wird nicht die Errichtung einer Netzinfrastruktur durch ein privates Unternehmen gefördert. Vielmehr erfolgt der Glasfaserausbau bis in die Gebäude durch eine öffentlich-rechtliche Körperschaft (derzeit noch kommunale Gebietskörperschaften) bzw. ein öffentliches Unternehmen. Die Bezeichnung als Betreibermodell ist insoweit irreführend, als nicht der Betrieb der Netzanlage das Ziel ist, sondern deren bloße Errichtung. Der Betrieb soll dann durch ein privates Unternehmen sichergestellt werden, das dazu das Netz vom Eigentümer pachtet. Im Idealfall bildet die Diensteerbringung eine dritte Ebene. Der eigentliche (private) Wettbewerb findet dann in zwei Stufen statt: In einer ersten Stufe um den Betrieb des Netzes - hier lässt sich die Wettbewerbsentscheidung nur durch eine Vergabeentscheidung als Instrument des Wettbewerbs bei knappen Gütern sicherstellen, verbunden mit der Befristung der Betriebsausübung. Auf einer zweiten Stufe findet dann ein Wettbewerb der Diensteerbringer um die Versorgung der Endkunden statt. Dazu besteht ein diskriminierungsfreier Zugang zum Netz als Voraussetzung der Diensteerbringung.

Dieses Ideal eines dreistufigen Modells (Eigentum/Betrieb/Diensteerbringung) wird in der bisherigen Praxis im Bundesgebiet in dieser Ausprägung zwar noch nicht umgesetzt. In den gegenwärtigen Betreibermodellen übernimmt im Regelfall ein Telekommunikationsdienstleister auch den Betrieb und verbindet damit Betrieb und Dienstleistungserbringung. Hierbei muss allerdings sichergestellt werden, dass Wettbewerbern ein Zugang zum Netz möglich ist. Aus der Schlüsselrolle des Betreibers entsteht in diesem Fall wiederum ein Regulierungsbedarf zugunsten der Mitbewerber. Aber immerhin beweist diese Umsetzungspraxis, dass ein Netzausbau in Verantwortung der öffentlichen Hand möglich und sinnvoll ist.

Der Vorteil dieses Vorgehens beim Netzausbau liegt auf der Hand: Die mit öffentlichen Mitteln errichtete Infrastruktur verbleibt als Wert in öffentlicher Hand. Es besteht aus der Verpachtung der physischen Infrastruktur sogar die Möglichkeit zur teilweisen Refinanzierung der Investition, insbesondere da langfristig mit stetig steigenden Anforderungen an die Infrastruktur auch die Zahlungsbereitschaft für Glasfaseranschlüsse zunehmen wird. Aller- 
dings wird dies derzeit noch durch die Vorgabe im Förderprogramm eingeschränkt, dem Markt den Erwerb des Netzes nach einem Mindestbetriebszeitraum anbieten zu müssen. Bleibt ein Kaufinteresse aus, besteht aber schon heute die Möglichkeit, das Netz im Eigentum einer öffentlich-rechtlichen Gebietskörperschaft zu belassen.

Dieses erprobte Modell kann durchaus der Schlüssel für ein effizienteres, schnelleres und zielorientierteres Vorgehen beim Ausbau konvergenter Netze aus Glasfaser und Mobilfunk werden. Für den Einstieg ist nicht einmal ein grundsätzlicher Richtungswechsel erforderlich. Im bestehenden Förderprogramm müsste lediglich eine Möglichkeit zur Förderung eines bundeseigenen Unternehmens aufgenommen werden, das die Errichtung der Netze - und zwar konvergenter Netze aus Mobilfunk- und Kabelnetzen - in weißen Flecken und in Zukunft auch in sogenannten grauen Flecken ${ }^{7}$ übernimmt. Weiterhin würde die geplante Förderung von Mobilfunkmasten in die bestehende Breitband-Richtlinie integriert, sodass weder eine eigene Verwaltungsvorschrift noch gegebenenfalls eine weitere Bewilligungsbehörde notwendig würde.

Ein solcher durch einen öffentlichen Träger ausgeführter Ausbau der physischen Infrastruktur - Glasfaseranschlüsse und Mobilfunkmasten (Schuler, 2019) - dürfte beihilferechtlich unproblematisch sein, da er bereits jetzt in einem Bundesland durch die EU-Kommission genehmigt ist (BMVI, 2015; Freistaat Bayern, 2018). Zwar wird der Staat dadurch zu einem Teilnehmer im Infrastrukturwettbewerb. Allerdings wird der Wettbewerb auch auf der Ebene der Infrastruktur durch ein solches Vorgehen nicht unterbunden und auf Diensteebene eher gestärkt. Denn durch die Neutralisierung des Faktors Übertragungsnetz werden die Ausgangsbedingungen der Marktteilnehmer bei der Diensteerbringung angeglichen.

\section{Zentralisiertes Engagement}

Einzuschränken ist jedoch, dass die Fokussierung auf den Ausbau in weißen und grauen Flecken das Problem fragmentierter Netztopografie nicht löst, sondern eher verschärft. Und für einen neuen Infrastruktureigentümer in solchen Gebieten stellt die Anbindung an Backhaulund Backbone-Netze eine besondere Herausforderung dar, die gegebenenfalls auch ein relevanter Kostenfaktor beim Netzausbau ist. Hier könnte es geboten sein, beim Ausbau in weißen Flecken nicht strikt das Ausbauvorhaben auf diese unversorgten Gebiete, die häufig aus nicht zusammenhängenden Straßen bzw. Wohngebieten

7 Als graue Flecken werden Gebiete angesehen, in denen lediglich ein NGA-Netz verfügbar und absehbar durch Marktteilnehmer kein weiteres NGA-Netz geplant ist. bestehen, zu begrenzen, sondern zusammenhängende Ausbaugebiete zu bilden, auch wenn dies graue Flecken oder gar ausreichend erschlossene Gebiete mit umfasst. Aus beihilferechtlichen Gründen muss dann umso mehr sichergestellt werden, dass der Einsatz von Fördermitteln nur für den Ausbau der weißen Flecken erfolgt. Die notwendigen Mittel zum Ausbau im übrigen Areal müssten zu marktüblichen Konditionen eingesetzt werden, gegebenenfalls unter Beteiligung privater Investoren. ${ }^{8}$

Der Beginn eines zentralisierten Engagements der öffentlichen Hand bei der Errichtung von Telekommunikationsnetzen ermöglicht aber nicht nur die Planung und Ausführung konvergenter und bedarfsgerecht optimierter Gigabitnetze. Eine vom Bund getragene Netzgesellschaft kann auch dazu eingesetzt werden, die langfristige Ausbauplanung zwischen Regionen übergreifend zu koordinieren. Zwar gibt es heute bereits im Bundesförderprogramm Breitband die Möglichkeit für Kommunen, sich einmalig Beratungsleistungen für eine Ausbauplanung durch externe Berater mit bis zu 50.000 Euro bezuschussen zu lassen. Allerdings erzeugt dies lediglich ein Flickenteppich an idiosynkratrischen, an den Grenzen von Gebietskörperschaften orientierten Planungen, die zudem noch ohne tatsächlichen praktischen Nutzen bleiben, da diese weitgehend losgelöst von den tatsächlichen Planungen, Netzbeständen und wirtschaftlichen Interessen der Unternehmen erfolgen.

Darüber hinaus hilft eine zentrale, indikative ${ }^{9}$ Planung eine effiziente Steuerung von Mitverlegungen durch die öffentliche Hand bei Tiefbauvorhaben vorzunehmen und damit das bisher nur zurückhaltend eingesetzte Instrument der Mitverlegung zu einer wichtigen Säule beim Netzausbau zu machen. Durch diese Kombination von Planung und Ausbau wurde dann zudem eine unmittelbare und exakt georeferenzierte Kartografierung der Netze möglich. So könnte eine solche Gesellschaft zu einem umfassenden Anbieter von georeferenzierten Infrastrukturdaten in Deutschland werden, der die bestehenden Wissenslücken zur Breitband- und Mobilfunkversorgung schließt.

Eine derart ausgestaltete Infrastrukturgesellschaft des Bundes ist wettbewerbspolitisch unbedenklich, wenn nicht sogar vorteilhaft (Kaffenberger, 2019). Auch wenn das hier vorgeschlagene zentralisierte Betreibermodell auf den ersten Blick in den Markt der Telekommunikationsdienstleis-

8 Vgl. die „Amsterdam Entscheidung“ bezüglich öffentlicher Investitionen in ein Glasfasernetz von 2007 (Europäische Kommission, 2007).

9 Auch wenn naturgemäß eine solche Planung ex ante nicht abschlieBend erfolgen kann, lassen sich wesentliche Faktoren einer Netzarchitektur wie der Zugang zum Backbone-Netz, Standorte der PoP (Point of Presence) sowie besondere Bedarfe, insbesondere potenzielle Mobilfunkmaststandorte, festlegen. 
tungen eingreift, bietet es doch gerade für die Telekommunikationsunternehmen wettbewerbsbezogene Vorteile. Denn zum einen können Verträge und Prozesse zur Nutzung von Infrastruktur zur Dienstleistungserbringung standardisiert und auch als Kostenfaktor berechenbarer gemacht werden. Zum anderen aber werden die Telekommunikationsunternehmen von den Kapitalkosten des Netzausbaus entlastet. Bisher finanzieren die Unternehmen den Ausbau vor. Das ist nicht nur einer der Gründe für den schleppenden Umstieg auf Glasfaserleitungen, sondern treibt angesichts des Umfangs der Programme auch die Verschuldungsquoten für Share- und Stakeholder in zumindest bedenkliche Höhen. Dieses für den Netzauf- und -ausbau gebundene Kapital steht den Unternehmen dann nicht für Investitionen in neue Geschäftsmodelle und Dienste zur Verfügung.

Im Ergebnis stellt sich damit eine ganzheitliche Infrastrukturgesellschaft für Glasfaser- und Mobilfunkausbau als bessere Alternative zum bisherigen Vorgehen dar. Wird der Bund künftig im Rahmen der von inm bereitgestellten Förderung selbst aktiv, ohne sich der Kommunen als Maßnahmenträger zu bedienen, wäre dies auch mit Blick auf die Bundeskompetenz in Art. 87 f GG durchaus konsequent. Soweit die Bundesregierung aber an der bisherigen Strategie trotz begrenzter Erfolge in der Umsetzung festhält, sollten die Länder über ein solches Modell nachdenken. Der Bund sollte dies ermöglichen, mindestens indem er die bisher zwingende Vorgabe im Förderprogramm, nur kommunale Gebietskörperschaften als Maßnahmenträger von geförderten Netzausbauvorhaben anzuerkennen, zugunsten von Landesgesellschaften erweitert.

\section{Literatur}

BMVI (Bundesministerium für Verkehr und digitale Infrastruktur) (2019a), Mobilfunkstrategie der Bundesregierung, https://www.bmvi.de/ SharedDocs/DE/Anlage/DG/Digitales/Mobilfunkstrategie.pdf? blob=publicationFile (6. Dezember 2019).

BMVI (2019b), Förderung zur Unterstützung des Breitbandausbaus in der Bundesrepublik Deutschland, Fassung der Richtlinie vom 28. November.

BMVI (2015), Rahmenregelung der Bundesrepublik Deutschland zur Unterstüt-zung des Aufbaus einerflächendeckendenNext Generation Access (NGA)-Breitbandversorgung.
Bundesnetzagentur (2018), Jahresbericht 2018, 56, https://www.bundesnetzagentur.de/SharedDocs/Mediathek/Jahresberichte/JB2018. pdf?_blob=publicationFile\&v=6 (12. Februar 2020).

Europäische Kommission (1995), Grünbuch über die Liberalisierung der Telekommunikationsinfrastruktur und der Kabelfernsehnetze vom 25. Oktober 1995, Teil I, Kom (94) 440 endg.

Europäische Kommission, (2007), Staatliche Beihilfen: Investitionen der Stadt Amsterdam in Glasfasernetz keine staatliche Beihilfe, https:// ec.europa.eu/commission/presscorner/detail/de/IP_07_1889 (13. Februar 2020).

Forsthoff, E. (1938), Die Verwaltung als Leistungsträger.

Freistaat Bayern (2018), Richtlinie zur Förderung des Ausbaus der Mobilfunkversorgung im Freistaat Bayern.

Grüne Bundestagsfraktion (2020), Mobilfunk als Daseinsvorsorge, Drucksache, 19/16518, Antrag vom 15. Januar, dip21.bundestag.de/ dip21/btd/19/165/1916518.pdf (13. Februar 2020).

GSMA Spectrum (2017), Effective Spectrum Pricing in Europe: Policies to support better quality and more affordable mobile services, https:// www.gsma.com/spectrum/wp-content/uploads/2017/10/EffectiveSpectrum-Pricing-in-Europe.pdf (12. November 2019).

Ilgmann, C. (2019), Breitbandausbau in Deutschland: eine strategische Analyse, Wirtschaftsdienst, 99(2), 120.

Kaffenberger, B. (2019), Das Netz muss neutral sein, https://www.zeit.de/ digital/internet/2019-02/breitbandausbau-digitalisierung-glasfasernetz-infrastruktur-internet-bundesregierung (20. März 2020).

Kleine Anfrage der Abgeordneten Margit Stumpp, Oliver Krischer, Matthias Gastel, weiterer Abgeordneter und der Fraktion BÜNDNIS 90/DIE GRÜNEN (2018) - Bundestags-Drucksache 19/6114 , 7. Juni.

OECD (2018), OECD Broadband statistics Dezember 2018, http://www. oecd.org/sti/broadband/broadband-statistics (6. Dezember 2019).

Open Signal (2018), https://www.opensignal.com/reports/2018/10/germany/mobile-networks-update (11. März 2019).

O.V. (2018), Beim Handynetz schlechter als Albanien, Welt, 27. Dezember, https://www.welt.de/politik/deutschland/article186160422/Mobilfunk-Studie-Deutsches-Netz-schlechter-als-das-in-Albanien.htm (28. Januar 2020).

P3 communications (2018), 5G National Roaming, https://www.gruenebundestag.de/fileadmin/media/gruenebundestag_de/themen_az/ medien/pdf/181210_Studie_Mobilfunk__Roaming.pdf (3. März 2020).

Rizzato, F. (2019), Understanding why so many German smartphone users are still 3G-only, https://www.opensignal.com/2019/11/07/understanding-why-so-many-german-smartphone-users-are-still-3gonly (28.Januar 2020).

Scheuer, S. (2019), Deutsche Kunden haben kaum Interesse an Glasfaseranschlüssen, Handelsblatt. 1. August, https://www.handelsblatt.com/ politik/deutschland/netzausbau-deutsche-kunden-haben-kauminteresse-an-glasfaseranschluessen/24860716. html?ticket=ST2633597-ykJNvyH6AWrDiewz3kR9-ap2 (28. Januar 2020).

Schuler, A. (2019), Die geplante Mobilfunkinfrastrukturgesellschaft: Zulässigkeit, Herausforderungen und offene Fragen, Netzwirtschaften \& Recht, 6, 269-274.

statista (2019), Anteil der Smartphone-Nutzer in Deutschland in den Jahren 2012 bis 2018, https://de.statista.com/statistik/daten/studie/585883/umfrage/anteil-der-smartphone-nutzer-in-deutschland/ (9. Juli 2020).

WIK und Umlaut (2019), Abschlussbericht zur Versorgungs- und Kostenstudie Mobilfunk im Auftrag des BMVI, 19. November.

Title: Telecommunication Networks in Germany - Expanding with a Public Company

Abstract: The broadband network in Germany still meets current demand. However, the quality of the existing infrastructure offers little incentive for private investors to adapt their infrastructure to future needs. Hence, the expansion of fibre optic lines is progressing too slowly. Government subsidy programmes create additional problems. A strategic reset is needed as the future will be a convergent system of fibre optic lines and mobile networks. The authors propose that the restructuring of the telecommunications infrastructure be entrusted to a state-owned infrastructure company. It should plan and construct nationwide fibre optic and mobile networks in a coordinated manner, based on a coherent analysis of demand and supply. Competition will then take place at the level of network operation and the provision of communication services.

JEL Classification: H54, D42, R58 